\title{
TRATAMIENTO MÉDICO NUTRICIONAL EN LA MUJER EMBARAZADA CON DIABETES
}

\author{
MEDICAL NUTRITION THERAPY \\ IN PREGNANT WOMEN WITH DIABETES
}

\author{
María Belén Tapia', Solange Houssay ${ }^{1,2}$, Guadalupe Vanoli1,2, Julio César Bragagnolo ${ }^{1,3}$ \\ Revisor: Jorge Alvariñas ${ }^{4}$
}

\section{RESUMEN}

Introducción: se ha observado un incremento en el diagnóstico de diabetes gestacional (DG) en mujeres embarazadas a nivel mundial durante los últimos años, siendo la prevalencia estimada de $14 \%$ a nivel global y de $10,36 \%$ en Argentina según el criterio diagnóstico de la Asociación Latinoamericana de Diabetes (ALAD). El plan de alimentación adecuado y el cambio en el estilo de vida asociados al automonitoreo de la glucemia capilar (AMGC) constituyen pilares fundamentales para alcanzar el objetivo glucémico en dos tercios de los casos de DG. A pesar de su importancia y de su recomendación en la práctica clínica, existe información científica limitada respecto de cuál es la prescripción alimentaria óptima para mejorar los resultados tanto en la madre como en el niño.

Objetivos: analizar el componente nutricional en el tratamiento de la mujer embarazada con diabetes y conocer cuáles podrían ser las recomendaciones para lograr los objetivos mencionados a través del análisis de la evidencia científica actual. La información proveniente de estudios randomizados y controlados es heterogénea, pero de dichos estudios surge que la intervención temprana con dieta, ejercicio y consejos sobre cambios de estilo de vida durante el primer trimestre o al inicio del segundo puede disminuir el riesgo de DG. El tratamiento médico nutricional en DG se basa en el control de la cantidad y la distribución de los hidratos de carbono para obtener el control glucémico óptimo sin hipoglucemias ni cetosis. El otro componente esencial es la ingesta calórica para la adecuada ganancia de peso.

Conclusiones: dada la creciente prevalencia de exceso de peso en la población general y en especial en las mujeres en edad fértil, deberían realizarse estudios donde ponderar la intervención con cambios del estilo de vida (dieta y actividad física) en este particular sector poblacional. Deberían elaborarse guías basadas en la evidencia donde se evalúe el impacto de los componentes de la dieta e ingesta calórica total como factores para mejorar los resultados materno-feto-neonatales.

Palabras clave: dieta; nutrición; embarazo; diabetes.

Revista de la Sociedad Argentina de Diabetes 2020; Vol. 54 (52-66)

\begin{abstract}
Introduction: in recent years, there has been a worldwide increase in the diagnosis of gestational diabetes (GD) in pregnant women, with the estimated prevalence of GD being $14 \%$ globally and 10,36\% in Argentina according to the Latin American Diabetes Association (LADA) diagnostic criteria. An adequate dietary plan in association with a healthy lifestyle change and glucose self-monitoring, are the cornerstones to achieve the glycemic goal in two thirds of GD cases.

Despite its importance and being broadly advised in clinical practice, there is limited scientific information regarding the optimal dietary prescription to improve results for both mother and child. Objectives: to analyze the nutritional aspects of the treatment of GD and to investigate what the recommendations would be to achieve the aforesaid outcomes, through the analysis of current scientific evidence.

Although there is heterogenous evidence from randomized controlled trials, it comes out from these studies that early intervention with diet, exercise and advice on healthy lifestyle during the first trimester or early in the second trimester, may decrease the risk of GD.

Nutritional treatment in GD is based on the control of the quantity and distribution of carbohydrates to attain optimal glycemic control without hypoglycemia or ketosis. Caloric intake is as well important for proper weight gain.

Conclusions: given the increasing prevalence of excess weight in the general population and especially in women of childbearing age, clinical trials should be carried out to study the results of interventions with lifestyle changes (diet and physical activity) in this special population. Evidence-based guidelines should be developed evaluating the impact of diet components and total caloric intake as factors to improve maternal-fetus-neonatal outcomes.
\end{abstract}

Key words: diet; nutrition; pregnancy; diabetes.

Revista de la Sociedad Argentina de Diabetes 2020; Vol. 54 (52-66)
1 Cátedra Nutrición, Facultad de Medicina, Universidad de Buenos Aires (UBA), Hospital Asociado Dr. José María Ramos Mejía, Ciudad Autónoma de Buenos Aires, Argentina

2 Médica de Planta, Hospital Dr. José María Ramos Mejía, Ciudad Autónoma de Buenos Aires, Argentina

3 Jefe de Unidad, Nutrición y Diabetes, Departamento de Medicina, Hospital Dr. José María Ramos Mejía, Ciudad Autónoma de Buenos Aires, Argentina

4 Médico Consultor, Nutrición, Hospital Dr. Enrique Tornú, Asesor del Comité de Diabetes y Embarazo, Sociedad Argentina de Diabetes, Ciudad Autónoma de Buenos Aires, Argentina
Contacto del autor: Julio César Bragagnolo

E-mail: jcbraga@intramed.net

Correspondencia: Hospital Dr. José María Ramos Mejía,

Gral. Urquiza 609, (C1221ADC), Ciudad Autónoma de

Buenos Aires, Argentina

Fecha de trabajo recibido: 02/03/20

Fecha de trabajo aceptado: 30/04/20

Conflictos de interés: los autores declaran que no existe conflicto de interés 


\section{INTRODUCCIÓN}

Las mujeres que desarrollan diabetes mellitus (DM) durante el embarazo tienen aumento del riesgo de padecer complicaciones fetales, maternas y neonatales a corto y largo plazo ${ }^{1,2}$. El hijo de madre con DM tiene mayor riesgo de macrosomía, y también aumenta el riesgo de obesidad, DM2, síndrome metabólico y trastornos del espectro autista (TEA) en el largo plazo ${ }^{3,4}$. En general, el mal control metabólico de la diabetes en el embarazo también se asocia con riesgo de aborto espontáneo, anomalías fetales, preeclampsia, hipoglucemia e hiperbilirrubinemia neonatales, entre otros ${ }^{5,6}$. El tratamiento médico nutricional es el pilar en la terapéutica de la mujer embarazada con diabetes junto con la actividad física. El plan de alimentación adecuado y el cambio en el estilo de vida asociados al automonitoreo de la glucemia capilar (AMGC) permiten alcanzar el objetivo glucémico en dos tercios de los casos de diabetes gestacional (DG) ${ }^{7}$. Sin embargo, a pesar de su importancia y de su amplia recomendación en la práctica clínica, existe información científica limitada respecto de cuál es la prescripción alimentaria óptima para mejorar los resultados tanto en la madre como en el niño.

\section{OBJETIVOS}

El objetivo de este trabajo es analizar el aspecto nutricional del tratamiento de la mujer embarazada con diabetes y conocer cuáles podrían ser las recomendaciones para lograr los objetivos mencionados a través del análisis de la evidencia científica actual.

\section{Criterios diagnósticos}

Se define como diabetes pregestacional (DPG) a la mujer con DM1 o DM2 que se embaraza, o a la embarazada que cumple con los criterios de diagnóstico de diabetes de la Organización Mundial de la Salud (OMS) en el primer trimestre del embarazo:

- Síntomas clásicos de diabetes (polidipsia, poliuria, polifagia y pérdida de peso) y una glucemia al azar mayor o igual a 200 mg/dl (sin relación con el tiempo transcurrido desde la última ingesta).

- Glucemia en plasma venoso en ayunas mayor o igual a $126 \mathrm{mg} / \mathrm{dl}$ confirmada con un segundo test realizado durante la semana.

- Glucemia en plasma venoso mayor o igual a $200 \mathrm{mg} / \mathrm{dl}$ a las 2 horas (h) de una carga de $75 \mathrm{~g}$ de glucosa durante una prueba de tolerancia oral a la glucosa (PTOG).
La DG se define como la disminución de la tolerancia a la glucosa de severidad variable que comienza o se diagnostica en el presente embarazo ${ }^{8,9}$.

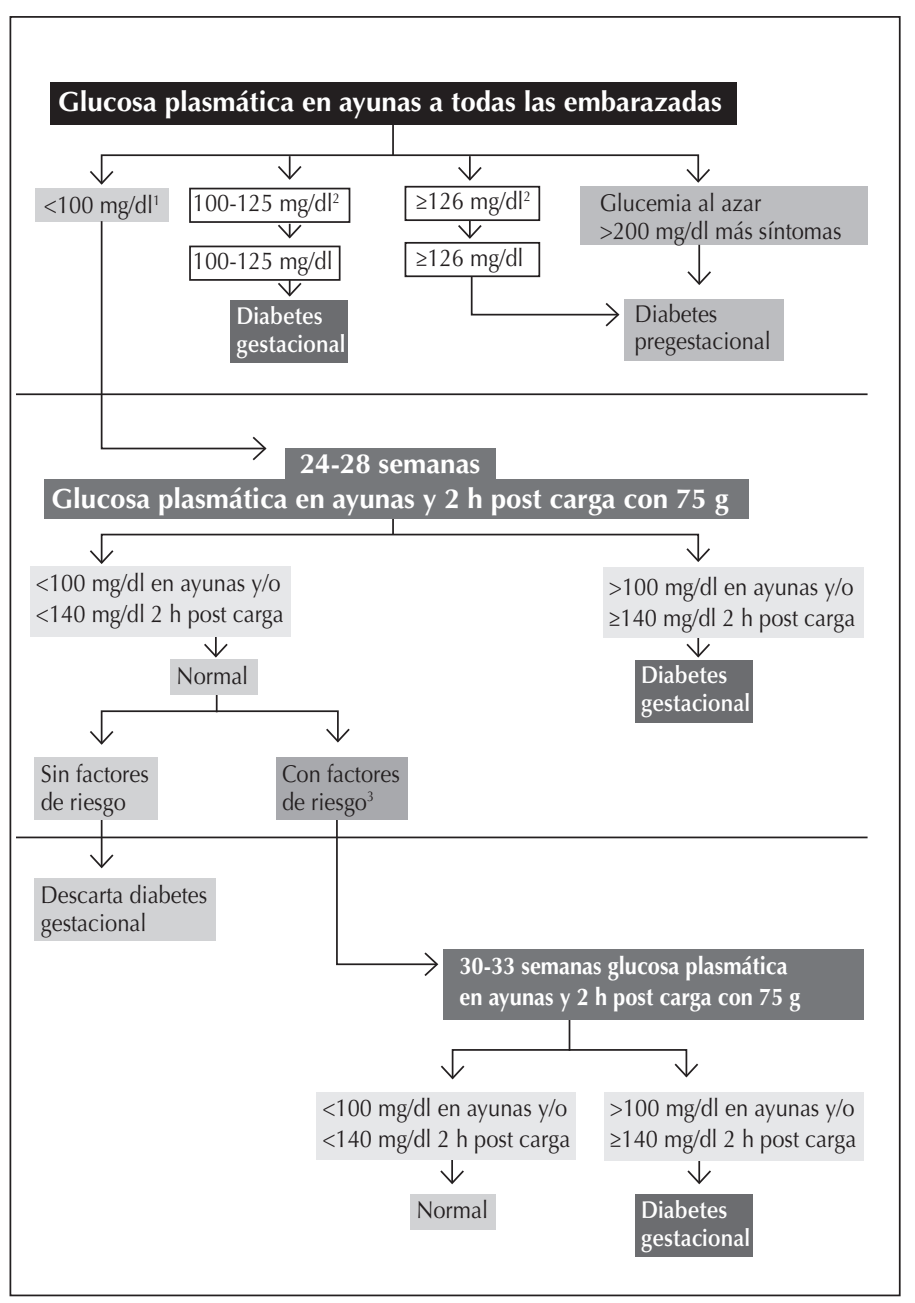

Fuente: Salzberg S, Alvariñas J, López G, et al. Guías de diagnóstico y tratamiento de diabetes gestacional. ALAD 2016. Rev ALAD 2016; 6:155-69.

' Según criterio médico y dependiendo de los recursos sanitarios e institucionales, se recomienda que, en pacientes con varios factores de riesgo de alto impacto para desarrollo de $D G$ y glucemia en ayunas normal, solicitar una p75 al inicio para descartar una DPG no diagnosticada. En caso de resultar normal, seguir el algoritmo establecido (C).

2 Repetir glucemia sin restricción alimentaria en un plazo máximo de siete días.

3 Ideal: retestear entre las 31 y 33 semanas a todas las embarazadas con factores de riesgo, y priorizar a aquellas que presenten factores de riesgo aparecidos o desarrollados durante el embarazo.

Gráfico 1: Algoritmo diagnóstico de diabetes gestacional (adaptado de las Guías de diabetes y embarazo del Ministerio de Salud de Chile, 2015). 
Si bien en nuestro medio adherimos a los criterios diagnósticos mencionados, debemos aclarar que existen controversias ${ }^{10}$. Entre éstas, el estudio $H y$ perglycemia and Pregnancy Outcome (HAPO) fue un trabajo internacional, observacional prospectivo, multicéntrico y doble ciego, con una población de 25.500 embarazadas, cuyo objetivo fue evaluar el riesgo de complicaciones maternas y feto-neonatales asociado a los niveles de glucosa materna. Se evidenció una relación lineal continua entre la glucemia materna y las complicaciones (sin punto de corte definido) ${ }^{11}$.

La International Association of Diabetes and Pregnancy Study Groups (IADPSG) estableció por votación, y con baja calidad de evidencia, una recomendación débil según la OMS, que toma como punto de corte el nivel glucémico en el cual las tasas de eventos fetales aumentan 1,75 veces sobre la media de la población del estudio, sin participación de centros de Latinoamérica ${ }^{12}$.

La Guía del National Institute for Health and Care Excellence 2015 (NICE, 2015) coincide con la propuesta de la ALAD 2007, de la Sociedad Argentina de Diabetes 2008 y de la ALAD 2016, que se utiliza como criterio diagnóstico en países diversos.

\section{Epidemiología}

En los últimos años hubo un incremento global en la prevalencia de obesidad y DM2. En la población argentina, de acuerdo con datos de la Cuarta Encuesta Nacional de Factores de Riesgo (ENFR) realizada por la Secretaría de Gobierno de la Salud y el Instituto Nacional de Estadística y Censos (INDEC), se observó que el 61,6\% de los argentinos tiene exceso de peso: $36,2 \%$ con sobrepeso y $25,4 \%$ con obesidad. El incremento en la prevalencia de DG parece tener múltiples causas aparentes que incluyen la prevalencia de obesidad, particularmente en la juventud (debido a los bajos niveles de actividad física y altos niveles de ingesta calórica), y el aumento de la sobrevida de las niñas nacidas con pesos al nacer extremos respecto del rango normal ${ }^{13}$, siendo el antecedente del peso al nacer un factor de riesgo muy importante en el desarrollo de DG ${ }^{14}$.

La prevalencia estimada de DG es de $14 \%$ o uno cada siete nacimientos a nivel global ${ }^{15}$. En España la prevalencia estimada es del $8,8 \%{ }^{16}$. Con respecto a los datos en Argentina, se determina una prevalencia del $10,36 \%$, según criterio diagnóstico de $A L A D$, mientras que con el criterio IADPSG asciende al 26,7\% ${ }^{17,18}$.

\section{Objetivos glucémicos en el embarazo}

El embarazo en mujeres con tolerancia normal a la glucosa se caracteriza por presentar niveles de glucemia en ayunas menores que fuera del embarazo debido a la captación de glucosa no dependiente de insulina por parte del feto y la placenta. Existe tendencia al aumento de la glucemia postprandial y eventual intolerancia a la glucosa como resultado de la acción de las hormonas placentarias.

En pacientes con diabetes preexistente, el objetivo glucémico usualmente se alcanza con el tratamiento médico nutricional y la administración de insulina. Debido a que el objetivo glucémico en el embarazo es más estricto, resulta de fundamental importancia que las embarazadas con diabetes ingieran una cantidad de hidratos de carbono suficiente, fraccionada y adecuada al valor calórico individualizado, que permita ajustar las dosis de insulina. Puede considerarse la utilización, según disponibilidad, del monitoreo continuo de glucosa (MCG) al aumentar el porcentaje de tiempo dentro del rango glucémico objetivo ( $T I R$, por sus siglas en inglés time in range) y minimizar el tiempo por debajo (TBR, por sus siglas en inglés time below range) o por encima del rango objetivo (TAR, por sus siglas en inglés time above range). Estos parámetros adquieren relevancia en pacientes con DM1. Un consenso reciente recomienda TIR $>70 \%$ entre $63-140 \mathrm{mg} / \mathrm{dl}$, TBR $<4 \%$ y $<1 \%$ con límites en 63 y 54 mg/dl respectivamente y un TAR $<25 \%$ sobre $140 \mathrm{mg} / \mathrm{dl}$. Se ha demostrado que el uso de MCG en DM1 durante el embarazo se relaciona con una leve mejoría en la $\mathrm{HbA} 1 \mathrm{c}$ sin mayor hipoglucemia y con la reducción del riesgo de recién nacidos grandes para edad gestacional (RNGEG), macrosomía, hipoglucemia neonatal y cuidados intensivos neonatales. No existe evidencia suficiente para determinar objetivos en MCG en mujeres con DG o con DPG y DM2 ${ }^{19}$.

El tratamiento médico nutricional debe estar a cargo de un profesional especialista en nutrición que establezca el plan alimentario y determine cuál es la ganancia de peso adecuada.

Los primeros meses del embarazo constituyen un período de aumento en la sensibilidad a la insulina, que implica la presencia de menores valores glucémicos y requerimientos menores de insulina en mujeres con DM1. Esta situación es seguida por el estado de insulinorresistencia que aumenta progresivamente durante el segundo y la primera parte del tercer trimestre. 
En las mujeres con función pancreática normal, la producción de insulina resulta suficiente para mantener los niveles normales de glucosa. Sin embargo, en aquellas con DG o diabetes preexistente aparecerá hiperglucemia de no mediar el tratamiento apropiado.

Teniendo en cuenta la fisiología, se recomienda el monitoreo de la glucosa en ayunas y en el período postprandial para seleccionar el tratamiento adecuado, verificar su efectividad, guiar su progresión y lograr la meta glucémica en las embarazadas con diabetes. El logro de los objetivos terapéuticos de la glucemia postprandial se asocia a un mejor control glucémico y a un menor riesgo de preeclampsia ${ }^{20}$.

De la misma manera que el Colegio Americano de Obstetras y Ginecólogos (ACOG), la Asociación Americana de Diabetes (ADA) recomienda que los objetivos glucémicos, tanto para las mujeres con DM1 o DM2 como para aquellas con DG son los siguientes ${ }^{12,13}$ : glucemia en ayunas menor a $95 \mathrm{mg} / \mathrm{dl}(5,3 \mathrm{mmol} / \mathrm{L}), 1$ hora (h) postprandial menor a $140 \mathrm{mg} / \mathrm{dl}(7,8 \mathrm{mmol} / \mathrm{L})$ o 2 h postprandial menor a $120 \mathrm{mg} / \mathrm{dl}(6,7 \mathrm{mmol} / \mathrm{L})^{21}$.

En el último consenso de la ALAD se recomienda utilizar el monitoreo glucémico como el parámetro más importante de control metabólico durante el embarazo, dado que permite cambiar conductas rápidamente, y su utilidad se incrementa con la educación y supervisión del cumplimiento de los objetivos glucémicos. Los objetivos terapéuticos son: glucemia en ayunas $70-90 \mathrm{mg} / \mathrm{dl}$, $1 \mathrm{~h}$ postprandial $85-140 \mathrm{mg} / \mathrm{dl}$ y $2 \mathrm{~h}$ postprandial 80-120 mg/dl, siendo fundamental evitar las hipoglucemias ${ }^{18,22}$.

\section{Tratamiento médico nutricional de la diabetes en mujeres embarazadas}

Las recomendaciones para su adecuado manejo incluyen:

- Educación sobre su diabetes. Resulta de importancia que se realice educación de la paciente sobre el manejo adecuado de su patología ya sea durante la consulta o en talleres especializados a tal fin. El período gestacional es una oportunidad para promover conductas saludables que repercutirán favorablemente a corto y largo plazo 23,17.

- Cambios en el estilo de vida. Luego de realizado el diagnóstico, la terapéutica debe iniciarse con el tratamiento médico nutricional, recomendaciones de actividad física y manejo adecuado de la ganancia ponderal (según el peso pregestacional).
A pesar de la heterogeneidad de los estudios randomizados y controlados, existe evidencia que sugiere que el riesgo de DG puede disminuirse con dieta, ejercicio y consejos sobre cambios de estilo de vida, especialmente cuando la intervención se inicia durante el primer trimestre o al inicio del segundo ${ }^{17}$.

Según la población, los estudios sugieren que aproximadamente el $70-85 \%$ de las mujeres diagnosticadas con DG con el criterio de CarpenterCoustan o National Diabetes Data Group (NDDG) pueden controlarla sólo con la modificación del estilo de vida, y podría ser una proporción mayor si se utilizara el criterio diagnóstico de la IADPSG ${ }^{24}$.

\section{Características del plan alimentario}

Los requerimientos nutricionales son los mismos que los que tienen las gestantes no diabéticas, teniendo en cuenta la distribución de los macronutrientes y especial cuidado en la selección alimentaria.

\section{Energía}

El costo metabólico total del embarazo es de aproximadamente $80.000 \mathrm{Kcal}$, cifra que proviene de investigaciones que estimaron los diferentes componentes materno-fetales involucrados en la actividad metabólica de mujeres europeas, bien nutridas y que presentaron adecuada ganancia de peso. En base a estas observaciones y estudios longitudinales que midieron el gasto energético a lo largo de la gestación, el Institute of Medicine (IM) propone un incremento en el requerimiento diario de $340 \mathrm{Kcal}$ en el segundo trimestre y de $452 \mathrm{Kcal}$ en el tercer trimestre. Como es poco probable que en los países periféricos o con economías emergentes se logre la atención prenatal desde el comienzo del embarazo, podría estimarse un aumento de $360 \mathrm{Kcal}$ en el segundo trimestre y de $475 \mathrm{Kcal}$ en el tercero, valores que son aplicables para su referencia sólo a nivel poblacional y no individual.

Por otro lado, según lo expresado por el documento de la Organización de las Naciones Unidas para la Agricultura y la Alimentación (Food and Agriculture Organization, FAO), se sugiere aumentar $250 \mathrm{Kcal}$ las necesidades previas de la mujer en el segundo trimestre y $500 \mathrm{Kcal}$ en el tercer trimestre. En todos los casos, debe tenerse en cuenta que las necesidades basales son sumamente variables de una mujer a otra dado que dependen de factores como la composición corporal pregestacional, el 
patrón de aumento de peso y fundamentalmente de condicionantes como el estatus hormonal de cada una. El gasto extra se determina por la actividad física que realiza la gestante ${ }^{25}$.

Las guías de la ALAD recomiendan agregar $300 \mathrm{Kcal}$ a partir del segundo trimestre. En caso de embarazadas con obesidad no es necesario adicionar calorías, pero debe tenerse en cuenta que el valor calórico total (VCT) no debe ser menor a $1.600 \mathrm{Kcal}$ y no menos de $160 \mathrm{~g}$ de hidratos de carbono en el día en forma fraccionada ${ }^{26}$. En cuanto al fraccionamiento, hay que asegurar seis porciones con adecuada distribución de los hidratos de carbono complejos con un mínimo de $15 \mathrm{~g}$ en cada ración, de las cuales una porción debe ser la colación nocturna. En caso de embarazo gemelar, sumar $450 \mathrm{Kcal}$ desde el segundo trimestre y en caso de embarazo múltiple, sumar $450 \mathrm{kcal}$ desde el primer trimestre. En cualquier caso, es importante adecuar el plan de alimentación según la curva de peso 27.

\section{- Ganancia de peso adecuada durante el embarazo}

La ganancia de peso excesiva en etapas tempranas del embarazo se correlacionó con mayor incremento del riesgo de desarrollo de DG. En un estudio retrospectivo, donde se evaluó la relación de los patrones de ganancia de peso y los niveles de glucosa en pacientes cumpliendo dieta, se concluyó que las pacientes adherentes a la dieta mantuvieron la DG bien controlada y ganaron menos peso luego del diagnóstico que las tratadas con insulina. Esto significa que el aumento de peso luego del diagnóstico de DG disminuye asociado al adecuado control glucémico ${ }^{28}$.

Existen recomendaciones de la ALAD y del Ministerio de Salud de la Nación en las cuales se indica la magnitud de ganancia ponderal en el embarazo de acuerdo con el peso de inicio (Tabla 1, Gráfico 2) 29,30.

El gráfico del Ministerio de Salud debe interpretarse como ganancia de peso adecuada: cuando la curva se encuentra entre \pm 1 desvío estándar (DE) (zona sombreada, normal). Ganancia de peso baja: cuando la ganancia de peso se ubica por debajo de -1 DE. Una ganancia de peso baja aumenta el riesgo de tener un niño de bajo peso. Ganancia de peso elevada: cuando la ganancia de peso se encuentra por encima del +1 DE aumenta el riesgo de tener un niño con alto peso al nacer. Por encima de +2 DE se considera que la embarazada tiene obesidad grado II. Si durante las sucesivas visitas se observa un cruce hacia arriba o debajo de los límites (o líneas), se sugerirán intervenciones oportunas como educación alimentaria para asegurar una ganancia de peso dentro de los parámetros normales. Si una mujer inicia el embarazo con sobrepeso (sector por encima del área sombreada), la ganancia total esperable es de entre 5 y $9 \mathrm{~kg}$, que representa la ganancia para el feto, la placenta y los anexos. Esto hará que en las sucesivas visitas prenatales la curva en la gráfica se acerque progresivamente al área sombreada (normal). Sin embargo, dado que no es aconsejable el descenso de peso (ya que podría poner en riesgo al feto), esta aproximación a la normal se realizará en forma lenta y progresiva sin registrarse pérdidas de peso.

\begin{tabular}{|l|c|}
\hline $\begin{array}{c}\text { Estado nutricional previo (según } \\
\text { índice de masa corporal, IMC) }\end{array}$ & $\begin{array}{c}\text { Ganancia de peso recomendada } \\
\text { en todo el embarazo }(\mathbf{k g})\end{array}$ \\
\hline Bajo peso (menos de 18,5$)$ & $12,5-18$ \\
\hline Peso normal $(18,5-24,9)$ & $11-16$ \\
\hline Sobrepeso $(25-29,9)$ & $7,0-11$ \\
\hline Obesidad $(\geq 30)$ & $5-7$ \\
\hline
\end{tabular}

Fuente: Salzberg S, Alvariñas J, López G, et al. Guías de diagnóstico y tratamiento de diabetes gestacional. ALAD 2016. Rev ALAD 2016; 6:155-69. Adaptado de Calvo et al. y Rasmussen et al.

Tabla 1: Recomendaciones de ganancia ponderal en el embarazo según estado nutricional al inicio.

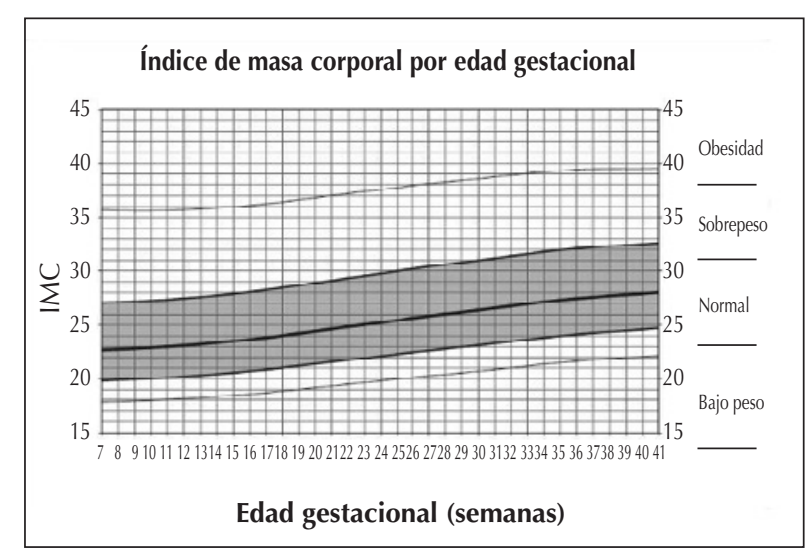

Fuente: Ministerio de Salud de la Nación. Nutrición y embarazo. Recomendaciones en Nutrición para los equipos de salud. Dirección Nacional de Maternidad e Infancia. Buenos Aires: Ministerio de Salud, 2012.

Gráfico 2:Índice de masa corporal por edad gestacional. 


\section{Macronutrientes \\ - Carbohidratos}

Se recomienda cubrir el $45-55 \%$ del VCT a predominio de carbohidratos $(\mathrm{HC})$ complejos, acompañados de fibra y distribuidos convenientemente a lo largo del día a fin de evitar ayunos prolongados ${ }^{25}$.

Diariamente la embarazada con peso normal necesita un mínimo aporte de glucosa en el orden de $175 \mathrm{~g}$ para satisfacer las demandas de oxidación en los propios tejidos, las necesidades fetales y evitar la cetosis. El feto es altamente sensible a las modificaciones de la glucemia materna. Cuando se mantienen dietas que elevan la respuesta glucémica, se estimula el hiperinsulinismo fetal, que a su vez condiciona un incremento en las vías anabólicas como la lipogénesis, responsable del alto peso al nacer y/o macrosomía fetal, y a largo plazo se asocia con mayor adiposidad en la infancia y mayor riesgo de síndrome metabólico en la adolescencia. A su vez, condiciona en la madre valores más elevados de glucemia en ayunas ${ }^{31,32}$.

En embarazadas con obesidad, las guías ALAD resaltan que más allá de fijar el VCT a no menos de $1.600 \mathrm{Kcal}$, los HC no deben ser menores a $160 \mathrm{~g}$ por día para evitar cetosis.

\section{- Proteínas}

El desarrollo fetal saludable depende de la disponibilidad de proteínas que proporcionan los componentes básicos necesarios para la formación del sistema nervioso central, músculo, colágeno, enzimas y anticuerpos. El colágeno es la matriz de soporte para la piel, los huesos, los vasos sanguíneos y otros tejidos corporales. Durante el embarazo, la madre debe tener un consumo adecuado de proteínas para satisfacer las necesidades del feto en crecimiento y sus propias necesidades progresivas en relación con su cambio corporal.

Se aconseja un aporte de proteínas de $1,1 \mathrm{~g}$ por kilo de peso corporal teórico o bien adicionar $25 \mathrm{~g}$ extra a las necesidades previas al embarazo. La FAO aconseja $30 \mathrm{~g}$ extra diarios en el tercer trimestre. Las proteínas deben proveer entre el 15 y $20 \%$ de la energía total, debiendo cubrirse no menos del $50 \%$ con proteínas de alto valor biológico ${ }^{33,34}$.

\section{- Lípidos}

Se debe cubrir entre el 30 y $35 \%$ del VCT; los ácidos grasos poliinsaturados omega 3: eicosapentaenoico (EPA) y especialmente el docosahexanoico (DHA), que es vital para la neurogénesis en el feto. La FAO recomienda DHA porque se necesita para el desarrollo de la retina y sus- tancia gris, estimula a los receptores de dopamina y serotonina, se requiere en la señalización de receptores de membranas celulares y nucleares, posibilita cambios en la fluidez y permeabilidad de las membranas celulares, y es el sustrato para la síntesis de mediadores antiinflamatorios.

Su necesidad aumenta en el tercer trimestre y cobra importancia sobre todo en mujeres multíparas que pueden tener reservas tisulares más bajas de DHA. La conversión endógena de ácido linolénico a DHA estaría aumentada en el embarazo. Las revisiones sistemáticas recientes concluyen que en las mujeres que consumen pescado de una a dos veces por semana se observa un beneficio en el desarrollo neurológico del niño, por lo que se enfatiza incluirlo en la alimentación, y evitar los pescados de gran tamaño y de mayor masa muscular que son los que pueden contener mayor cantidad de metilmercurio (que es producto de la contaminación en las plataformas marítimas). La FAO estima un aporte necesario promedio de $600 \mathrm{~g}$ de ácidos grasos esenciales durante toda la gestación, es decir, 2,2 g/día. Estas necesidades se satisfacen con una alimentación equilibrada que provea al menos un $20 \%$ del contenido energético diario como grasas, y no hay evidencia de que los requerimientos de grasa total o como porcentaje de la energía difieran en relación con la indicación fuera del embarazo ${ }^{31,35}$.

Para las embarazadas o lactantes se establece una ingesta mínima para una salud óptima y un desarrollo del feto y el lactante adecuados de $300 \mathrm{mg}$ diarios de EPA más DHA, de los cuales al menos $200 \mathrm{mg}$ diarios deben corresponder al $\mathrm{DHA}^{36}$.

El valor superior para la ingesta de EPA+DHA se establece en $2 \mathrm{~g}$ diarios debido a las evidencias experimentales que indican que los suplementos altos de LCPUFA n-3 pueden incrementar la peroxidación lipídica y reducir la producción de citoquinas. Sin embargo, la Comisión de Expertos reconoce que valores más altos de consumo, tales como $3 \mathrm{~g}$ diarios, reducen otros riesgos cardiovasculares y no producen efectos adversos a corto plazo o plazo intermedio en ensayos aleatorios, y que algunos individuos en poblaciones con un gran consumo de animales marinos ingieren cantidades mayores sin evidencia aparente de efectos perjudiciales ${ }^{37}$.

En un metaanálisis de Cochrane se observó la reducción de parto prematuro $<37$ semanas y parto prematuro temprano $<34$ semanas en las pacientes que recibieron ácidos grasos omega 3. 
Se detectó una probable reducción en el riesgo de muerte perinatal y de ingreso a cuidados neonatales, y una reducción en el riesgo de recién nacidos con bajo peso al nacer, incluso posiblemente un ligero aumento en el riesgo de recién nacidos grandes para edad gestacional ${ }^{37}$.

\section{- Fibra}

La inclusión de fibra dietética ayuda a controlar los niveles de glucosa e insulina, e influye favorablemente en los niveles de lípidos en plasma en pacientes con diabetes. La fibra soluble se caracteriza por su capacidad de disolverse en agua y formar geles viscosos, lo que permite que el tiempo de tránsito intestinal disminuya, y se retrase la absorción y la digestión completa de nutrientes. Además favorece la disminución de las excursiones de glucosa postprandiales, la reducción de la insulinemia y los niveles de lípidos en suero. Algunos de los principales tipos de fibra soluble son: $\beta$-glucanos, inulina, fructoligosacáridos, arabinoxilanos y pectinas. El consumo de fibra insoluble puede actuar como una barrera física, por lo que podría reducir la velocidad de absorción de los productos digestivos a través del ribete en cepillo del enterocito. Uno de los mecanismos de acción para la fibra dietética y los prebióticos es la fermentación en el colon y los cambios favorables en la microbiota intestinal. Los prebióticos son ingredientes alimenticios no digeribles que estimulan selectivamente el crecimiento y/o la actividad de un número limitado de bacterias colónicas y, posteriormente, mejoran la salud del huésped. Una dieta adecuada en fibras también reduce la frecuencia de constipación y hemorroides, situaciones comunes en el embarazo. La recomendación general de ingesta de fibra en pacientes con diabetes en el embarazo es de 20 a 25 g/ día ${ }^{38,39,40}$.

\section{Micronutrientes \\ - Hierro}

El IM estableció la ingesta de referencia en $23 \mathrm{mg}$ diarios de requerimiento promedio estimado y en $27 \mathrm{mg}$ diarios de recomendación dietética. Se recomienda dar a todas las embarazadas suplementos de hierro en forma de tabletas. Considerando que en el tercer trimestre de gestación gran parte de las embarazadas puede tener depósitos insuficientes de hierro, y que la prevalencia de anemia es del 30\%, la normativa del Ministerio de Salud de la Nación estableció la provisión de 60 mg de hierro elemental y $400 \mu \mathrm{g}$ de ácido fólico desde el primer control del embarazo $32,28,41$.
Según las recomendaciones de la OMS, para prevenir la anemia materna, la sepsis puerperal, el bajo peso al nacer y el nacimiento prematuro se recomienda que las embarazadas tomen un suplemento diario por vía oral${ }^{42}$.

Las distintas sales ferrosas disponibles en el mercado presentan mínimas diferencias respecto de su absorción (tasa 10-15\%) y contienen cantidades variables de hierro elemental.

Hay varios factores que deben tenerse en cuenta sobre el tratamiento con sulfato ferroso. El sulfato ferroso debe administrarse lejos de las comidas dado que muchos alimentos disminuyen la absorción de hierro (hasta en un 40 a 50\%) debido a la formación de complejos poco solubles. Algunas de las sustancias que inhiben la absorción de hierro son calcio, fosfatos, fitatos y fenoles, mientras que entre los que la facilitan se incluyen a la vitamina $\mathrm{C}$, los citratos y el ácido clorhídrico.

La biodisponibilidad del hierro elemental varía según el tiempo de tratamiento. Al comenzarlo, la absorción es de aproximadamente un 13,5\% y después de 20-30 días disminuye a 5\%. La absorción varía con la severidad de la anemia y con la coexistencia con otras enfermedades. La eficacia del tratamiento depende de su tasa de absorción, condicionada por la cantidad, posología, estado de los depósitos biológicos, actividad eritropoyética y factores intraluminales que interfieren en su absorción ${ }^{41}$ (ver Anexo con preparados disponibles).

La principal desventaja del hierro oral reside en la elevada frecuencia de efectos adversos gastrointestinales (10-40\%), en forma de dolor abdominal, pirosis, náuseas y vómitos, estreñimiento o diarrea, con tasas de incumplimiento terapéutico mayores al 10-20\%.

\section{- Ácido fólico}

El IM considera que una ingesta diaria de $600 \mu \mathrm{g}$ de folato dietético equivalente (FDE) se necesita para mantener las concentraciones normales de folatos en el glóbulo rojo, por lo cual se recomienda su suplementación en el embarazo ${ }^{26}$. Durante este período esta vitamina es indispensable para acompañar la rápida división celular que se produce, como así también para reducir el riesgo de anemia. Durante los primeros 28 días del embarazo esta vitamina es fundamental para reducir hasta en un $75 \%$ el riesgo de malformaciones del cierre del tubo neural. A nivel individual, se recomienda el uso preconcepcional de 0,4 mg/día de ácido fólico para prevenir la ocurrencia de defectos 
del tubo neural. Se aconsejan $4 \mathrm{mg} / \mathrm{día}$ de ácido fólico para prevenir la recurrencia en mujeres con antecedentes de niños con malformaciones del tubo neural y la suplementación con 0,5 mg de ácido fólico durante el segundo y tercer trimestre ${ }^{30,41}$.

Las formulaciones comerciales de nuestro país se presentan en comprimidos de $1 \mathrm{mg}, 5 \mathrm{mg}$ y $10 \mathrm{mg}$.

\section{- Calcio y vitamina D}

La evidencia sugiere que si se mantienen las ingestas de calcio recomendadas para la mujer no embarazada en el orden de los 1.000-1.300 mg diarios, la masa ósea materna no se utilizaría para cubrir las necesidades fetales, por lo que no se aconseja indicar el uso rutinario de suplemento de calcio. En mujeres con ingestas inadecuadas y factores de riesgo, la administración diaria de un suplemento que contenga de 1.000 a $2.000 \mathrm{mg}$ de calcio se asocia con una menor prevalencia de preeclampsia ${ }^{43}$. En relación con la vitamina $\mathrm{D}$, independientemente de su efecto en la absorción y metabolismo óseo, se sugirió que su suplementación durante la gestación podría reducir el riesgo de preeclampsia, bajo peso al nacer y parto prematuro, aunque las evidencias aún resultan controversiales ${ }^{44}$. La recomendación de vitamina D para mujeres embarazadas es de 600 unidades internacionales diarias.

En relación con el déficit de vitamina $D$, cuatro metaanálisis (que incluyeron de seis a 31 estudios observacionales) encontraron un aumento significativo en el riesgo de diabetes gestacional (Aghajafari 2013; Christesen 2012; Poel 2012; Wei 2013), mientras que solo uno (con dos estudios) no encontró asociación significativa (Thorne-Lyman 2012) (5 $^{5}$.

Tanto la OMS como las guías dietéticas recientes de los Estados Unidos no recomiendan explícitamente un suplemento de vitamina $D$ durante el embarazo, excepto para las personas con una exposición limitada a la luz solar o quienes usan protector solar $21,26,27$.

Se necesitan mayores evidencias de ensayos controlados aleatorizados para evaluar prospectivamente los efectos de la administración de suplementos de vitamina D durante el embarazo, en particular en relación con el riesgo de eventos adversos maternos.

\section{- Colina}

Es un componente de los fosfolípidos, precursor de la síntesis de lecitina, que integra a las membranas celulares. La disponibilidad de colina es fundamental para el desarrollo del sistema ner- vioso central fetal y en la producción de acelticolina. Existe evidencia de su efecto sobre la función cognitiva de los niños. Las embarazadas deben ingerir 450 mg/día proveniente de huevo, carne, pescado, fuentes vegetales (porotos), repollitos de bruselas, brócoli y espinaca ${ }^{46}$.

\section{- Sodio}

Según la recomendación de las guías de la $A L A D$, en relación con el cloruro de sodio, no debe indicarse menos de $5 \mathrm{~g}$ por día (2 g de sodio/día). Se restringirá mínimamente en caso de hipertensión ${ }^{17}$.

En una revisión sistemática publicada por Cochrane, donde se evaluó el efecto de la restricción de sodio en mujeres normotensas frente a otro grupo de mujeres a las que se les aconsejó continuar con dieta habitual, no se observaron diferencias estadísticamente significativas en preeclampsia, muerte perinatal, admisión a cuidados críticos y caída del score APGAR a los minutos de nacimiento entre ambos grupos. La OMS no recomienda la restricción dietaria de sodio durante el embarazo ${ }^{47}$.

\section{- Zinc}

La ingesta insuficiente de zinc se asocia con bajo peso al nacer y parto prematuro. Durante el embarazo existe mayor absorción intestinal. Algunas madres tienen mayor riesgo de deficiencia cuando realizan dietas altas en fibras, tienen bajo consumo de calcio o hierro, y presentan alteraciones en la absorción como puede ocurrir en pacientes que se sometieron a cirugía bariátrica. Se sugiere hacer énfasis en el consumo de alimentos ricos en zinc como carnes, vísceras, huevos y cereales integrales ${ }^{25,33}$.

\section{- Edulcorantes de alta intensidad}

En mujeres embarazadas con DG, que eligen consumir edulcorantes de alta intensidad, deben seleccionar sólo aquellos aprobados por la Administración de Alimentos y Drogas (Food \& Drug Administration, FDA) de EE.UU., y para limitar su consumo a la ingestión diaria admisible (IDA) establecida. La FDA aprobó la seguridad de seis edulcorantes de alta intensidad (sacarina, aspartamo, acesulfame, sucralosa, neotame y advantame) cuando se consumen dentro de la IDA por la población general, incluidas mujeres embarazadas. Glucósidos de esteviol y Luo Han Quo (extractos de fruta del monje) también se reconocen generalmente como seguros cuando se consumen dentro de la IDA $37,48,49$.

\section{- Alcohol}

Se debe reforzar la abstinencia del alcohol durante el embarazo para las mujeres con DG. Es la opción más segura para eliminar el riesgo de 
complicaciones relacionadas con el alcohol, como defectos de comportamiento o neurológicos, deficiencias de crecimiento, anomalías faciales y deterioro del desarrollo intelectual ${ }^{38}$.

\section{- Cafeína}

Algunos autores aconsejan la abstinencia total de cafeína durante el embarazo, mientras otros recomiendan un máximo de 300 mg/día durante la gesta ${ }^{51}$.

En algunos estudios se describió una correlación entre los altos niveles de cafeína en plasma materno y menor reactividad fetal, mayor variabilidad de la frecuencia cardíaca fetal y aumento de la frecuencia respiratoria fetal ${ }^{50}$.

\section{Estrategias de planificación alimentaria en DG}

El plan alimentario debe proveer una ingesta adecuada de calorías para promover la salud fetalneonatal y materna, alcanzar el objetivo glucémico y favorecer una ganancia de peso que sea apropiada. El requerimiento calórico no difiere entre embarazadas con o sin DG. El tratamiento médico nutricional en DG se basa en el control de la cantidad y la distribución de los hidratos de carbono para obtener el control glucémico óptimo sin hipoglucemias ni cetosis. El plan alimentario debe estar guiado por la ingesta dietaria de referencia (IDR). La IDR de macronutrientes para toda embarazada recomienda un mínimo de $175 \mathrm{~g}$ de hidratos de carbono (160 g en caso de obesidad), un mínimo de $71 \mathrm{~g}$ de proteínas y $28 \mathrm{~g}$ de fibra?. Dicho consumo debe distribuirse entre tres a cuatro comidas y dos a tres colaciones ${ }^{25}$. Como sucede en el plan alimentario de todo paciente con diabetes, la cantidad y tipo de carbohidratos impactará en los niveles de glucemia, particularmente en las excursiones glucémicas postprandiales. Aunque el control de la cantidad y el tipo de HC indicado es ampliamente utilizado para optimizar el control glucémico, existen además otros recursos que también son importantes en el manejo de DG, que incluyen la introducción de opciones de alimentos saludables, control de la porción y prácticas de preparación de los alimentos ${ }^{21,51}$.

Existen diferentes estudios que evaluaron diversas estrategias de planificación alimentaria en búsqueda de mejorar los resultados maternoneonatales en pacientes con DG. Con respecto a la dieta con bajo índice glucémico, se reportaron evidencias de su beneficio en pacientes con DM2.
El estudio de Moses de 2009 investigó si la dieta con hidratos de carbono de bajo índice glucémico podía reducir el número de mujeres con DG que requería insulina durante el embarazo. Se incluyeron 63 mujeres con DG que fueron aleatorizadas a recibir dieta con hidratos de carbono de bajo índice glucémico, o dieta convencional alta en fibra y alto índice glucémico. El grupo asignado a la dieta de bajo índice glucémico presentó una significativa disminución en la indicación de insulina, sin compromiso de los resultados obstétricos-fetales ${ }^{51,52}$.

También se compararon los efectos de la intervención con dieta de baja carga glucémica vs. una dieta control sobre el control glucémico, el metabolismo lipídico, la ganancia de peso materno y el peso de nacimiento. Se reclutaron y aleatorizaron 95 embarazadas y, como resultado, el grupo asignado a la dieta con baja carga glucémica tuvo mayor disminución en la glucosa de ayunas y postprandial, y menor aumento en el colesterol total y triglicéridos. No se hallaron diferencias significativas en la ganancia de peso corporal ni en el peso de nacimiento, $u$ otros resultados materno-fetales ${ }^{53}$.

En 2013 se realizó un trabajo que evaluó una población de 130 mujeres con DG pero esta vez la hipótesis fue si una dieta baja en contenido de hidratos de carbono (con 40\%) podría disminuir el requerimiento de insulinización respecto de un grupo con dieta control (con 55\% HC del VCT). El valor calórico de cada paciente se calculó en base al peso pregestacional, con un mínimo de $1.800 \mathrm{Kcal} / \mathrm{d}$ ía. Ambas dietas tenían contenido proteico similar (20\% del VCT) pero variaban en el contenido de grasa, siendo del $40 \%$ en el grupo de la dieta baja en $\mathrm{HC}$ y del $25 \%$ en el grupo control, principalmente a expensas de aumentar la ingesta de aceite de oliva. Como resultado, el requerimiento de insulinoterapia fue del $54,7 \%$ en ambos grupos ${ }^{54}$. No hay evidencia sobre cuál es la cantidad ideal de contenido de hidratos de carbono para el tratamiento de DG, y sólo existe como recomendación el consumo mínimo de 175 g/día en mujeres con peso norma ${ }^{54,55}$.

Con respecto al efecto de la Dietary Approaches to Stop Hypertension (dieta DASH) en mujeres con DG, se realizó un estudio randomizado y controlado con 52 mujeres con DG. Fueron asignadas a consumir dieta DASH y dieta control durante cuatro semanas. La dieta control contenía 45-55\% de HC, $15-20 \%$ de proteínas y $25-30 \%$ de grasas. La dieta DASH era rica en frutas, vegeta- 
les, granos enteros y productos de bajo contenido graso, con menor contenido de ácidos grasos saturados, colesterol y granos refinados, con un total de $2.400 \mathrm{mg} / \mathrm{dí}$ a de sodio. Se evaluaron requerimientos de insulinoterapia, necesidad de cesárea y prevalencia de polihidramnios. Como resultados, en el grupo que siguió la dieta DASH el 46,2\% requirió cesárea, mientras que en el grupo control fue de $80,8 \%(p=0,01)$. También hubo diferencia significativa en el porcentaje que requirió insulinoterapia (23\% para dieta DASH vs. $73 \%$ dieta control). Los hijos de madres con dieta DASH tuvieron menor peso al nacimiento, comparado con el grupo control $(3,222$ vs. 3,818 kg). La circunferencia cefálica y el índice ponderal también resultaron menores en aquellos nacidos de madres con dieta DASH ${ }^{56}$.

En 2016 se realizó un metaanálisis que incluyó cinco trabajos para determinar si las dietas con bajo índice glucémico reducen el riesgo de complicaciones maternas y neonatales en embarazadas con DG. También se intentó determinar cuál era la contribución de la fibra dietaria a los efectos de las dietas con bajo índice glucémico en mujeres embarazadas. Se evidenció que las dietas con bajo índice glucémico reducen el riesgo de macrosomía. Además, un subgrupo de análisis reportó que las dietas con bajo índice glucémico, con niveles de fibra dietaria aproximadamente equivalente a las dietas control, reducen el requerimiento de insulinoterapia, y que las dietas con bajo índice glucémico con contenido aumentado de fibra dietaria disminuyen el riesgo de macrosomía, más allá del efecto que logra la dieta con bajo índice glucémico en forma aislada ${ }^{53}$.

Recientemente se efectuó un metaanálisis, que incluyó 18 estudios, para determinar si la intervención con dietas modificadas (definidas como las intervenciones con dietas diferentes a las recomendaciones estándar indicadas al grupo control) en mujeres con DG ofrece mejores resultados en el control glucémico y/o resultados neonatales.

Se analizaron las diferencias en glucemia materna en ayunas, glucemia postprandial, HbA1c, HOMA-IR, necesidad de tratamiento con insulina o hipoglucemiantes orales, peso de nacimiento, macrosomía y alto peso para la edad gestacional. Los distintos tipos de intervención con dieta modificada incluyeron la de bajo índice glucémico $(n=4), D A S H$ $(n=3)$, bajo contenido en hidratos de carbono $(n=2)$, modificada en grasas $(n=2)$, enriquecida con proteína de soja $(n=2)$, restricción calórica $(n=1)$, alta en fibra $(n=1)$ y dietas étnicas $(n=1)^{57}$.
A pesar de la heterogeneidad entre los estudios, se encontró un efecto moderado de la intervención con dietas en los resultados sobre la glucemia materna, incluyendo cambios en la glucemia en ayunas, postprandial y el requerimiento de tratamiento con insulina, así como también en el peso de nacimiento neonatal. Sin embargo, la calidad de la evidencia es baja debido a las limitaciones en el diseño de los estudios incluidos, a la inconsistencia entre sus resultados y la imprecisión en la estimación de los efectos. Varios de los estudios son demasiado pequeños para demostrar diferencias significativas en los objetivos primarios. Siete de ellos tenían menos de 50 participantes y sólo dos tenían más de 100. La corta duración de la mayoría de las intervenciones dietarias y la edad gestacional tardía en la que se iniciaron podrían tener limitado impacto en los resultados glucémicos y en el peso de nacimiento. Es más, no puede concluirse si la mejoría en la glucemia materna y en el peso de nacimiento se deben a la reducción en la ingesta calórica, a la mejoría de la calidad de los nutrientes, o a cambios específicos en el tipo de hidratos de carbono y/o proteínas. Debe tenerse en cuenta también que la reducción en la ingesta de hidratos de carbono para disminuir la glucemia postprandial puede compensarse con un mayor consumo de grasas conduciendo potencialmente a efectos adversos en la insulinorresistencia materna y en la composición corporal fetal ${ }^{57}$.

La dieta mediterránea (DietMed) se reconoce como un patrón dietético saludable con múltiples beneficios para la salud y la prevención de diversas patologías. La relación entre la adhesión a la DietMed y la reducción de riesgo de desarrollar DM2 las analizaron múltiples ensayos epidemiológicos y clínicos que la avalan.

Sin embargo, hasta el momento son pocos los estudios que relacionan la DietMed con la disminución del riesgo de desarrollo de DG, y los mecanismos por los cuales el patrón de DietMed protege del desarrollo de DG no están totalmente aclarados.

Los beneficios para la salud de la DietMed se describieron inicialmente en los años 1950-60 en el "Estudio de los siete países", donde se relataba el papel de esta dieta en la enfermedad coronaria. Los hábitos alimentarios del área mediterránea llamaron la atención luego de que se constatara que, en los países bañados por el mar mediterráneo, con una alimentación basada en el consumo de 
aceite de oliva, frutas, cereales, verduras frescas y vino, la incidencia de enfermedades coronarias era significativamente menor que en otros países del norte de Europa, cuya alimentación incluía un alto consumo de cerveza, carnes rojas, fiambres, embutidos, chacinados y grasas ${ }^{58}$.

Las características más importantes de la DietMed son:

- Alto consumo de verduras, legumbres, frutas, frutos secos y cereales integrales.

- Alta ingesta de aceite de oliva, tanto para cocinar como para aderezar los alimentos.

- Baja ingesta de grasas saturadas.

- Moderado consumo de pescados, principalmente azules.

- Entre baja y moderada ingesta de productos lácteos y principalmente en forma de queso y yogur.

- Baja ingesta de carne y productos cárnicos procesados.

- Regular pero moderada ingesta de vino.

- Bajo consumo de cremas, manteca y margarina.

- Alto consumo de ajo, cebolla y especias.

Solamente en años recientes se publicaron datos enfocados específicamente en la relación existente entre el patrón de DietMed y el desarrollo de DG. Un estudio, desarrollado en 10 países mediterráneos, puso manifiesto una reducción de la incidencia de DG en gestantes con buena adherencia a un patrón de DietMed. La reducción en la incidencia de DG varió entre 34 a 38\% según los criterios utilizados para el diagnóstico de DG en aquellas pacientes situadas en el tercilo superior de adherencia a DietMed ${ }^{59}$.

En una cohorte australiana se comprobó de igual manera la reducción de DG incidente con la adherencia a la DietMed en un modelo ajustado, mientras que el patrón denominado "carnes, aperitivos y dulces" mostró una asociación con mayor riesgo de DG después de ajustar por factores de confusión (socioeconómicos, reproductivos y de estilo de vida ${ }^{60}$.

El grupo del Hospital San Carlos de Madrid realizó un ensayo controlado y aleatorizado prospectivo para comparar el efecto de dos tipos distintos de dieta. Se incluyeron a todas las embarazadas normoglucémicas (ayunas <92 mg/dl) que asistieron al centro en forma consecutiva entre la semana 8-12 de embarazo. Se asignaron al grupo intervención (GI) 500 embarazadas (GI: dieta mediterránea suplementada con aceite de oliva extra virgen y pistachos) y 500 al grupo control (GC) con dieta estándar limitada en grasa.
Hubo una reducción del riesgo relativo del criterio de valoración final, incidencia de DG en la semana 24-28, del 30\% en el GI comparado con el GC.

Las pacientes del $\mathrm{Gl}$ tuvieron glucemias en ayunas y a las $2 \mathrm{~h}, \mathrm{y}$ hemoglobina $\mathrm{A} 1 \mathrm{C}$ e índice HOMA significativamente menores en la semana 24-28. También menos mujeres en el GI requirieron insulina y hubo menor frecuencia de infecciones urinarias, cesáreas de urgencia, partos prematuros, traumas perineales, y bajo y elevado peso para edad gestacional ${ }^{61}$.

Si bien se postula que la DietMed tiene efecto antiinflamatorio e inmunomodulador (fenoles y aceite de oliva), resulta complicado y difícil separar los efectos de los distintos alimentos o comidas, pues no se comen nutrientes sino alimentos que los contienen en distintas proporciones, y a menudo patrones dietéticos ricos en un nutriente tienden a asociarse con un mayor o menor consumo de otros, siendo muy compleja su separación.

De hecho, las personas con alta adherencia a un patrón dietético mediterráneo no podrían consumir las elevadas cantidades de verduras y legumbres que ingieren, de no ser por su acompañamiento con aceite de oliva como aderezo en ensaladas, frituras y guisos.

El aceite de oliva es beneficioso porque es fuente de ácidos grasos monoinsaturados, reduce la glucemia postprandial (GPP) y tiene propiedades antiinflamatorias. Podría reducir la carga glucémica de las ingestas y alienta el consumo de verduras que lo acompañan en la cocina del sur del Europa ${ }^{62}$.

Se ha sugerido que el estrés oxidativo e inflamatorio puede influir como marcador de posibles vías involucradas en la patogenia de la DG. Los alimentos ricos en azúcar y grasas saturadas demostraron promover el estrés oxidativo y la inflamación, mientras que las verduras, legumbres, nueces y granos enteros son bajos en energía y grasa y tienen un alto contenido de fibra dietética, magnesio, vitamina $\mathrm{E}$ y otros antioxidantes que pueden contribuir a la reducción de los marcadores de estrés oxidativo y de inflamación. No está claro si el estrés oxidativo y la inflamación son los mecanismos que vinculan a la dieta con DG, si son independientes o parcialmente independientes del índice de masa corporal (IMC), o si es el aumento del porcentaje de grasa corporal y su localización el evento que media entre la dieta, los procesos de estrés oxidativo, la inflamación y la incidencia de DG63,64. 
El análisis de patrones dietéticos ofrece una oportunidad práctica y aplicable de intervención en salud pública, y tiene en cuenta las interacciones o efectos sinérgicos que pueden existir entre los distintos componentes de un determinado patrón dietético. Los beneficios demostrados de la DietMed, que es también una dieta de bajo índice glucémico, probablemente no se deban al efecto aislado de algún componente de la misma, sino al conjunto de todos ellos.

La DietMed incluye alimentos que se recomiendan habitualmente por considerarse saludables, no hay prohibiciones de alimentos, es una dieta sabrosa, variada y accesible que impulsa fundamentalmente el consumo de alimentos locales, y cuyo beneficio radica tanto en la variedad de alimentos que se incluyen como en las técnicas culinarias utilizadas para optimizar sus cualidades y la forma de consumirlos.

La DietMed protege de DM2 a pacientes que han padecido $D G$, en las que se ha demostrado una reducción del $40 \%$ del riesgo ${ }^{65}$.

Por lo tanto, la adherencia a la DietMed desde la programación del embarazo y durante el mismo, podría reducir el riesgo de DG al disminuir la susceptibilidad de las pacientes con factores de riesgo a desarrollarla. Asimismo, el mantenimiento de la adherencia a este patrón dietético podría influir sobre el eventual desarrollo posterior de DM2 en mujeres con diagnóstico previo de DG ${ }^{66}$.

\section{CONCLUSIONES}

El tratamiento médico nutricional resulta prioritario dado que la intervención a través de la dieta en el enfoque terapéutico integral de la embarazada con DPG o DG tiene un impacto favorable sobre la glucemia materna y el peso de nacimiento del recién nacido. A pesar de la baja calidad en la evidencia científica, su revisión resalta el rol clave que este tratamiento tiene en el manejo de la DG y aún su potencial mayor beneficio. Dada la prevalencia creciente de la DG, debe promoverse la realización de nuevos estudios para evaluar la potencial intervención a través de la alimentación y la actividad física en trabajos de investigación con mayor número de mujeres embarazadas o en edad fértil. Como la mayoría de las mujeres con DG inicia su embarazo con un IMC elevado, las recomendaciones basadas en la evidencia consideran que tanto los componentes de la dieta, así como la ingesta calórica total, son factores de importancia a considerar para optimizar los resultados maternofeto-neonatales y sentar las bases de la salud futura de la madre y el niño/a por nacer. Es en los países periféricos o con economías emergentes, como el nuestro, donde el impacto de las consecuencias de la DG tiene mayor envergadura y donde se requiere que las autoridades sanitarias destinen recursos que mejoren el estado nutricional de las mujeres en edad fértil con promoción de la educación en estilos de vida saludable que las pueda conducir a un embarazo con menor exposición a complicaciones para ellas y su descendencia.

\section{BIBLIOGRAFÍA}

1. Landon MB, Spong C, Thom E, et. al; for the Unice Kennedy Shriver National Institute of Child Health and Human Development Maternal-Fetal Medicine Units Network. A multicenter, randomized trial of treatment for mild gestational diabetes. $\mathrm{N}$ Engl J Med 2009; 361:1339-1348

2. Boney CM, Verna A, Tucker R, Vodir BR. Metabolic syndrome in childhood: association with birth weight, maternal obesity and gestational diabetes mellitus. Pediatrics 2005; Mar115(3): e290-e296.

3. Sacks KN, Friger M, Shiham-Vardi I, Abokaf H, et al. Prenatal exposure to gestational diabetes mellitus as an independent risk factor for long-term neuropsychiatric morbidity of the offspring. American Journal of Obstetrics and Gynecology 2016; 380.e1-380.e5

4. Xiang $A$, Wang $X$, Martínez $M$, Walthall J, et al. Association of maternal diabetes with autism. JAMA 2015; 313(14):1425-1434.

5. Holmes VA, Young IS, Patterson CC, et. al. Diabetes and preeclampsia intervention trial study group. Optimal glycemic control, pre-eclampsia and gestational hypertension in women with type 1 diabetes in the Diabetes and Pre-Eclampsia Intervention Trial. Diabetes Care 2011 Aug; 34(8):1683-8.

6. Dabelea D, Hanson RC, Lindsay RS, et al. Intrauterine exposure to diabetes conveys risks for type 2 diabetes and obesity: a study of discordant sibships. Diabetes 2000 Dec; 49(12):2208-11.

7. American Diabetes Association. Management of diabetes in pregnancy. Sec 13. In: Standards of Medical Care in Diabetes 2017. Diabetes Care 2017; 40(Suppl.1): S114-S119.

8. American Diabetes Association. Clinical practice recommendations gestational diabetes mellitus. Diabetes Care 2001; 24(Suppl.1): S77-S79.

9. Metzger BE, Coustan DR. Summary and recommendations of the Forth International Workshop-Conference on Gestational Diabetes Mellitus. The Organizing Committee. Diabetes Care 1998 Aug; 21 (Suppl 2): B161-7

10. Agarwal MM. Gestational diabetes mellitus: an update on the current international diagnostic criteria. World J Diabetes 2015; 6(6):782-791.

11. Metzger BE, Contreras M, Sacks DA, et al; Study Cooperative Research Group (HAPO). Hyperglycemia and adverse pregnancy outcomes. N Engl J Med 2008; 358:991-2002.

12. Diagnostic criteria and classification of hyperglycemia first detected in pregnancy. Geneva: World Health Organization; 2013 (WHO/NMH/MND/13.2). Disponible en: http://www.who. int/diabetes/publications/Hyperglycemia_Pregnancy. Acceso: $24 \% 5 / 2020$. 
13. Metzger BE, BuchananTh A, et al. Summary and recommendations of the Fifth International Workshop Conference on Gestational Diabetes Mellitus. Diabetes Care July 2007 Jul; 30(Suppl 2): S251-60.

14. Innes DE, Byers TE, Marshall JA, et al. Association of a woman's own birth weight with subsequent risk for gestational diabetes. JAMA 2002; 287: 2534-254.

15. Guanguata L, Linnenkap $U$, Beagley J, et al. Global estimates of the prevalence of hyperglycemia in pregnancy. Diabetes Res Clin Pract 2014; 103(2):176-185.

16. Ricart W, López J, Mozas J, et al. Potential impact of American Diabetes Association (2000). Criteria for diagnosis of gestational diabetes mellitus in Spain. Diabetologia 2005 Jun; 48(6):1135-1141.

17. Salzberg S, Alvariñas J, López $G$, et al. Guías de diagnóstico y tratamiento de diabetes gestacional. ALAD 2016. Rev ALAD 2016; 6:155-69.

18. Sucasini MR, Lewin ML, Aguera D, et al. Prevalencia de diabetes gestacional en Argentina y complicaciones materno-fetoneonatales según los distintos criterios diagnósticos. Reporte preliminar. Revista de la Sociedad Argentina de Diabetes 2014; 48:82-83.

19. Battelino T, DanneT, et al. Clinical targets for continuous glucose monitoring data interpretation: recommendations from the International Consensus on Time in Range. Diabetes Care Aug 2019; 42(8):1593-1603. Doi: 10.2337/dci19-0028.

20. Bain E, Crane M, Tieu J, et al. Diet and exercise intervention for preventing gestational diabetes mellitus. Cochrane Data Base Syst Rev 2015; 4.

21. Management of diabetes in pregnancy. Standards of Medical Care. En: Diabetes-2020. American Diabetes Association. Diabetes Care 2020 Jan; 43(Suppl 1): S183-S192.

22. Rovira G, Jawerbaum S, Glastein L. Recomendaciones para el tratamiento de pacientes con diabetes pregestacional. Sociedad Argetina de Diabetes. Grupo Latinoamericacno de Diabetes y Embarazo. ALAD 2018. Rev ALAD 2018;8.

23. Lapertosa $S$, et al. Educación terapéutica de mujeres con diabetes gestacional (EduGest). Revista de la Sociedad Argentina de Diabetes Vol. $53 \mathrm{~N}^{\circ} 3$ Suplemento septiembre-diciembre de 2019; 121-126.

24. Jovanovic C, Peterson L, Peterson CM, Reed GF, et al. Maternal postprandial glucose levels and infant birth weight. The diabetes in early pregnancy study. The National Institute of Child Health and Human development. Am J Obstet Gynecol 1991; 164:103-111.

25. Food and Nutrition Board, Institute of Medicine. Nutrition during pregnancy. Part 1: Weight gain. Washington DC: National Academy of Sciences 1990.

26. Food and Nutrition Board, Institute of Medicine. Nutrition during pregnancy. Part 2: Nutrient supplements. Washington DC: National Academy of Sciences 1990. Citas repetidas.

27. NICE, National Institute for Health and Care Excellence. Gestational diabetes, risk assessment, testing, diagnosis and management. Disponible en: http://pathways.nice.org.uk/pathways/ diabetes-in-pregnancy. Actualizado: 4/06/ 2019: 1-10. Acceso: 26/5/2020.

28. Dolatkhah N, Hajifaraji M. Nutrition therapy in managing women with gestational diabetes: literature review. Journal of Family and Reproductive Health 2018; Vol 12, N² 2 .

29. López L, Suárez M. Fundamentos de nutrición normal. Ed. Ateneo $2^{\circ}$ Edición 2017.
30. Ministerio de Salud De la Nación. Nutrición y embarazo. Recomendaciones en nutrición para los equipos de salud. Dirección Nacional de Maternidad e Infancia. Buenos Aires: Ministerio de Salud, 2012. Disponible en: www.msal.gob.ar embarazo y nutrición-Ministerio de salud. Acceso: 26/05/2020.

31. American Diabetes Association. Nutrition recommendations and intervention for diabetes: a position statement of de ADA. Diabetes Care 2008; 31 (suppl 1): 561-578.

32. Yamamoto J, Kellet J, Balsells M, Patterson A, Hadar E, Sola I, Gich I, et al. Gestational diabetes mellitus and diet: a systematic review and meta-analysis of randomized controlled trials examining the impact of modified dietary interventions on maternal glucose control and neonatal birth weight. Diabetes Care 2018; 41:1346-1361.

33. National Academy of Sciences, Ed. Dietary reference intake: energy, carbohydrate, fiber, fat, fatty acids, cholesterol, protein and aminoacids (macronutrients). Washington DC, National Academy Press 2005.

34. Girolami D, González-Infantino C. Clínica y terapéutica en la nutricion del adulto. Buenos Aires. Ed. El Ateneo, 2008.

35. Fats and fatty acids in human nutrition report of an expert consultation. FAO Food and Nutrition Paper 91, FAO, Rome, 2010.

36. Greemberg J, Bell S, et al. Omega-3 fatty acid supplementation during pregnancy 2008;1: 162-169

37. Middleton P, Gomersall JC, Gould JF, Shepherd E, Olsen SF, Makrides M. Omega-3 fatty acid addition during pregnancy. Cochrane Database of Systematic Reviews 2018; Issue 11. Art. $N^{\circ}$ : CD003402. Doi: 10.1002/14651858.CD003402.pub3.

38. Hull $\mathrm{H}$, Herman, Gibbs $\mathrm{H}$. The effect of high dietary fiber intake on gestational weight gain, fat accrual and postpartum weight retention: a randomized clinical trial. BMC pregnancy and childbirth 2020; 20:2013

39. Duarte-Gadea M, González-Pacheco D, Reader D, et al. Academy of Nutrition and dietetics gestational diabetes evidencebased nutrition practice guideline. Journal of Nutrition and Dietetics 2018; 2212-2672.

40. Kalkwarf $H$, Bell R, et al. Dietary fiber intake and insulin requirements in pregnant women with type 1 diabetes. Journal of the American Dietetic Association 2001; 101: 305-310.

41. Cereceda-Bujaico M, Quintana-Salinas $M$. Consideraciones para la adecuada alimentación durante el embarazo. Revista Peruana de Ginecololgía y Obstetricia 2014; 60(2):153-159.

42. Comité Nacional de Hematología. Sociedad Argentina de Pediatría. Anemia ferropénica. Arch Argent Pediatr 2001; 99(2):162-167.

43. Hofmeyr GJ, LawrieT, Duley L, Torloni MR. Calcium supplementation during pregnancy for preventing hypertensive disorders and related problems. Cochrane Database Syst Rev 2018 Oct 1;10(10):CD001059.

44. Regil L, Palacios C, Lombardo L. Vitamin D Supplementation for women during pregnancy. Cochrane Database of Systematic Reviews January 2016 Jan 14;(1):CD008873.

45. Palacios C, Trak-Fellermeier MA, Martínez RX, López-Pérez L, Lips P, Salisi JA, John JC, Peña-Rosas JP. Regimens of vitamin D supplementation for women during pregnancy. Cochrane Database of Systematic Reviews 2019; Issue 10. Art. N: CD013446.

46. Caudill MA. Prenatal and postnatal health: evidence of increased choline needs. J Am Diet Assoc 2010; 110:1198-206.

47. WHO recomendations for prevention and tretament of preclampsiaand eclampsia. Geneva: World Health organization; 2011. 
48. Tsirou E, Grammatikopoulou M, et al. Guidelines for medical nutrition therapy in gestational diabetes mellitus: systematic review and critical appraisal. Journal of the Academy of Nutrition and Dietetics 2019; 2212-2672.

49. Laviada-Molina H, Escobar-Duque I, Peryra E, et al. Consenso de la Asociación Latinoamericana de Diabetes sobre el uso de edulcorantes no calóricos en personas con diabetes. Rev ALAD 2018; 8:152:74.

50. Mulder EJ, Tegaldo $L$, et al. Foetal responde to maternal coffe intake: role of habitual versus nonhabitual caffein consumption. J Phsychopharmacol 2010; 24: 1641

51. Moses R, Barker M, Winter M, Petocz P, Brand-Miller J. Can a low-glycemic index diet reduce the need for insulin in gestational diabetes mellitus? Diabetes Care 2009; 32:996-1000.

52. Wen-Jun Ma, Zhi-Hong Huang, Bi-Xia Huang, Ben-Hua Qi, YanJun Zhang, et al. Intensive low-glycemic-load dietary intervention for the management of glycaemia and serum lipids among women with gestational diabetes: a randomized control trial. Public Health Nutrition 2015; 18(8):1506-1513.

53. Jenkins DJA, Kendall CWC, McKeown Eyssen G, Josse AR, Ngyuen TH, Corrigan S, Banach MS, Ares S, Mitchell S, Emam A, Augustin LSA, Parker TL, Leiter LA. Effect of a low-glycemic index or a high-cereal fiber diet on type 2 diabetes: a randomized trial. JAMA 2008; 300(23):2742-2753.

54. Moreno-Castilla $C$, Hernández M, Bergua $M$, Álvarez $M$, Arce $M$, et al. Low carbohydrate diet for treatment of gestacional diabetes mellitus. Diabetes Care 2013; 36: 2233-2238.

55. Wei J, Heng W, Gao J. Effect of low glycemic index diets on gestational diabetes mellitus. A meta-analysis of randomized controlled clinical trials. Medicine (Baltimore) 2016; 95(22): e 3792.

56. Asemi Z, Samimi M, Tabassi Z, Esmaillzadeh A.The effect of DASH diet on pregnancy outcomes in gestational diabetes: a randomized controlled clinical trial. Eur J of Clin Nutr 2014; 68(4):490-495.

57. Yamamoto J, Kellet J, Balsells M, Patterson A, Hadar E, Sola I, Gich I, et al. Gestational diabetes mellitus and diet: a systematic review and meta-analysis of randomized controlled trials examining the impact of modified dietary interventions on maternal glucose control and neonatal birth weight. Diabetes Care 2018; 41:1346-1361.
58. Keys A, Menotti A, Karvonen MJ, Aravanis C, Blackburn H, Buzina R, Djordjevic BS, Dontas AS, Fidanza F, Keys MH, Kromhout D, Nedeljkovic S, Punsar S, Seccareccia F, Toshima H. The diet and 15-year death rate in the seven countries study. Am J Epidemiol 1986; 124:903-915.

59. Karamanos B, Thanopoulou A, Anastasiou E, et al. Relation of the mediterranean diet with the incidence of gestacional diabetes. Eur J Clin Nutr 2014; 68: 8-13.

60. Schoenaker DA, Soedamah-Muthu SS, Callaway LK, Mishra GD. Pre-pregnancy dietary patterns and risk of gestational diabetes mellitus: results from an Australian population-based prospective cohort study. Diabetologia 2015; 58:2726-35.

61. Assaf-Balut C, García de la Torre N, Durán A, Fuentes M, Bordiú $E$, del Valle $L$, et al. A Mediterranean diet with additional extra virgin olive oil and pistachios reduces the incidence of gestational diabetes mellitus (GDM): A randomized controlled trial: The St. Carlos GDM prevention study. PLoS One 2017 Oct 19; 12(10):e0185873. Doi. org/10.1371/journal.pone.0185873

62. Trichopoulos D, Lagiou P. Mediterranean diet and cardiovascular epidemiology. Eur J Epidemiol 2004; 19:7-8.

63. López-García E, Schulze MB, Fung TT, et al. Major dietary patterns are related to plasma concentrations of markers of inflammation and endothelial dysfunction. Am J Clin Nutr 2004 80:1029-35.

64. Dai J, Jones DP, Goldberg J, et al. Association between adherence to the mediterranean diet and oxidative stress. Am J Clin Nutr 2008; 88:1364-70.

65. Tobias DK, Hu FB, Chavarro J, Rosner B, Mozaffarian D, Zhang C. Healthful dietary patterns and type 2 diabetes mellitus risk among women with a history of gestational diabetes mellitus. Arch Intern Med 2012; 172:1566-72.

66. Mozas-Moreno J, Gómez-Fernández J, Olmedo-Requena R, Jiménez-Moleón JJ. Dieta mediterránea y diabetes gestacional. Revisión. Nutr Clin Med 2016; X (1): 54-67.

\section{ANEXO}

\section{Vademecum de preparados medicinales con hierro}

\begin{tabular}{|c|c|c|c|c|}
\hline Droga & Nombre comercial & Forma de presentación & Equivalencia fe elemental & Vía \\
\hline \multirow[t]{4}{*}{ Sulfato ferroso } & Ibersol & Comprimidos & $105 \mathrm{mg}$ & VO \\
\hline & Siderblut & Comprimidos & $60 \mathrm{mg}$ & VO \\
\hline & Fefol & Comprimidos & $45 \mathrm{mg}$ & VO \\
\hline & Fefolic & Comprimidos & $105 \mathrm{mg}$ & VO \\
\hline \multirow[t]{2}{*}{ Fumarato ferroso } & Anemidox ferrum & Comprimidos & $105 \mathrm{mg}$ & VO \\
\hline & Ferretab & Comprimidos & $60 \mathrm{mg}$ & VO \\
\hline \multirow[t]{2}{*}{ Hierro polimaltosato } & Ferranin & Ampollas $\times 2 \mathrm{ml}$ & $1 \mathrm{ml}: 50 \mathrm{mg}$ & IM \\
\hline & Ferranin complex & Comprimidos & $100 \mathrm{mg}$ & VO \\
\hline Hierro sacarato & Ferranin IV & Ampollas & $1 \mathrm{ml}: 20 \mathrm{mg}$ & EV \\
\hline Hierro dextran & Yectafer & Amp $\times 2 \mathrm{ml}$ & $1 \mathrm{ml}: 50 \mathrm{mg}$ & IM \\
\hline \multirow[t]{2}{*}{ Hierro elemental } & Hierroquick 40 & Comprimidos & 1 comprimido: 40 mg & VO \\
\hline & Hierroquick 80 & Comprimidos & 1comprimido: $80 \mathrm{mg}$ & VO \\
\hline
\end{tabular}




\begin{tabular}{|c|c|c|c|c|}
\hline Droga & Nombre comercial & Forma de presentación & Equivalencia fe elemental & Vía \\
\hline \multirow[t]{5}{*}{ Sulfato ferroso } & Ibersol & Comprimidos & $105 \mathrm{mg}$ & VO \\
\hline & Siderblut & Comprimidos & $60 \mathrm{mg}$ & VO \\
\hline & Fefol & Comprimidos & $45 \mathrm{mg}$ & VO \\
\hline & Hemofer 200 & Comprimidos & $65 \mathrm{mg}$ & VO \\
\hline & Ferrofolic & Comprimidos & $105 \mathrm{mg}$ & VO \\
\hline \multirow[t]{3}{*}{ Fumarato ferroso } & Anemidox ferrum & Comprimidos & $105 \mathrm{mg}$ & VO \\
\hline & Hemoref 330 & Comprimidos & $108,9 \mathrm{mg}$ & VO \\
\hline & Ferretab & Comprimidos & $60 \mathrm{mg}$ & VO \\
\hline \multirow[t]{2}{*}{ Hierro polimaltosato } & Ferranin & Ampollas $\times 2 \mathrm{ml}$ & $1 \mathrm{ml}: 50 \mathrm{mg}$ & IM \\
\hline & $\begin{array}{r}\text { Ferranin complex } \\
\text { Siderblut poli } \\
\text { Vitalix } \\
\text { Rexfol } \\
\text { Tenvic }\end{array}$ & Comprimidos & $100 \mathrm{mg}$ & $\mathrm{VO}$ \\
\hline Hierro sacarato & $\begin{array}{r}\text { Ferranin IV } \\
\text { Dixafer IV } \\
\text { Ferive } \\
\text { Sucrox IV Complex } \\
\text { Venofer }\end{array}$ & Ampollas & $1 \mathrm{ml}: 20 \mathrm{mg}$ & $\mathrm{EV}$ \\
\hline Hierro dextran & $\begin{array}{l}\text { Yectafer } \\
\text { Ferinon } \\
\text { Intrafer }\end{array}$ & Ampollas $\times 2 \mathrm{ml}$ & $1 \mathrm{ml}: 50 \mathrm{mg}$ & IM \\
\hline \multirow[t]{2}{*}{ Hierro elemental } & Hierroquick 40 & Comprimidos & 1 comprimido: $40 \mathrm{mg}$ & VO \\
\hline & Hierroquick 80 & Comprimidos & 1 comprimido: $80 \mathrm{mg}$ & VO \\
\hline
\end{tabular}

VO: vía oral; EV: vía endovenosa; IM: vía intramuscular. 